\title{
CORRESPONDENCE
}

\section{Liver toxicity of sitaxentan in pulmonary arterial hypertension}

\section{To the Editors:}

Ambrisentan, bosentan and sitaxentan are endothelin receptor antagonist (ERA) drugs approved for the treatment of patients with pulmonary arterial hypertension $(\mathrm{PAH})$ and are included in the treatment algorithm of the 2009 pulmonary hypertension $(\mathrm{PH})$ guidelines of the European Society of Cardiology (ESC) and the European Respiratory Society (ERS) [1].

Hepatotoxicity is a frequent side-effect of ERA therapy, which is contra-indicated in patients with mild to severe hepatic impairment (Child-Pugh class B-C) and elevated serum aminotransferases prior to initiation of treatment. In addition, liver function testing on a monthly basis is recommended in $\mathrm{PAH}$ patients treated with these compounds, in order to detect an increase in the serum aminotransferases [1]. In most cases, liver injury is dose-related and reversible with dose reduction or drug discontinuation, suggesting that hepatotoxicity is caused by a dose-dependent toxic effect. The exact mechanisms by which ERA therapy causes liver injury are not clear. It has been suggested that ERAs or their metabolites competitively inhibit a bile salt transporter pump, leading to intracellular accumulation of bile salts, but other mechanisms cannot be excluded.

Seven cases of severe hepatitis-like drug reactions have been described in association with sitaxentan; two at a higher dose than currently approved (of whom one patient died from liver failure) [2], and five treated with the currently approved dose of $100 \mathrm{mg}$ once daily (of whom one patient died from liver failure, one underwent heart, lung and liver transplantation and one recovered after prednisone treatment; two recovered after standard treatment) [3-5].

Two additional cases of fatal liver injury related to the use of sitaxentan in PAH patients have been recently reported [6]. In all these cases, liver function deteriorated despite discontinuation of the drug. To our knowledge there are no reports in the medical literature of similar complications with bosentan or ambrisentan. In fact, no deaths related to liver toxicity have been reported for bosentan out of $\sim 80,000$ patients treated worldwide and for ambrisentan out of $\sim 10,000$ patients treated. In contrast, four deaths and one case of liver transplantation related to the use of sitaxentan have been observed among the $\sim 2,000$ patients treated worldwide. However, a warning label has been added to the US prescriber information of bosentan mentioning rare cases of liver failure in patients treated with bosentan.

It has been suggested that there are at least two types of liver injury associated with the use of ERA therapy [3]; one toxic, i.e. dose-dependent and reversible after dose reduction or discontinuation of the drug, the other possibly idiosyncratic or immunologically mediated. The former type of liver injury is by far more common and, almost invariably, has a benign clinical course. The second type appears to be rare but, at the same time, very dangerous, as it can result in severe liver failure.

The recent report of the two additional cases of fatal liver injury related to the use of sitaxentan in $\mathrm{PAH}$ patients enrolled in a randomised controlled study has prompted Pfizer to voluntarily discontinue all ongoing clinical trials with this compound and to withdraw the commercial drug (Thelin; $100 \mathrm{mg}$ sitaxentan tablets) from the market worldwide [6]. Thelin has been authorised in the European Union (EU) since 2006 for the treatment of PAH and has been marketed in $16 \mathrm{EU}$ member states, and in Australia and Canada.

It has been suggested by the European Medicines Agency [6] that patients taking Thelin or participating in Thelin studies are advised not to stop treatment and to consult their treating physician to review their treatment at their next scheduled appointment.

The task force of the ESC/ERS 2009 PH guidelines would like to make the following recommendations to the treating physicians.

1) For the time being, no PAH patient should start de novo therapy with Thelin.

2) For patients already on treatment with Thelin, transition to another ERA, such as bosentan (Tracleer) or ambrisentan (Volibris), should be considered.

3) In cases in which the PAH patient was treated with Thelin due to previous adverse reactions with Tracleer and Volibris, the transition to another class of approved PAH drugs should be considered (prostanoids or phosphodiesterase 5 inhibitors).

\section{N. Galiè*, M.M. Hoeper" ${ }^{\#}$, J.S.R. Gibbs ${ }^{\top}$ and G. Simonneau ${ }^{+}$ for the Task Force for the Diagnosis and Treatment of Pulmonary Hypertension of the European Society of Cardiology and the European Respiratory Society *Insititute of Cardiology, Bologna University Hospital, Bologna, Italy. "Dept of Respiratory Medicine, Hannover Medical School, Hannover, Germany. "National Heart \& Lung Institute, Imperial College London and Hammersmith Hospital, London, UK. ${ }^{+}$Dept of Pneumology and ICU, National Reference Centre for Pulmonary Hypertension, South Paris University, Hopital Antoine Béclère, Clamart, France.}


Correspondence: N. Galiè, Institute of Cardiology, University of Bologna, via Massarenti 9, 40138-Bologna, Italy. E-mail: nazzareno.galie@unibo.it

Statement of Interest: Statements of interest for all authors can be found at www.erj.ersjournals.com/site/misc/statements. xhtml

\section{REFERENCES}

1 Galiè N, Hoeper MM, Humbert M, et al. Guidelines for the diagnosis and treatment of pulmonary hypertension. Eur Respir J 2009; 34: 1219-1263.

2 Barst RJ, Rich S, Widlitz A, et al. Clinical efficacy of sitaxsentan, an endothelin-A receptor antagonist, in patients with pulmonary arterial hypertension: open-label pilot study. Chest 2002; 121: $1860-1868$.
3 Hoeper MM, Olsson KM, Schneider A, et al. Severe hepatitis associated with sitaxentan and response to glucocorticoid therapy. Eur Respir J 2009; 33: 1518-1519.

4 Lee W-TN, Kirkham N, Johnson MK, et al. Sitaxentan-related acute liver failure in a patient with pulmonary arterial hypertension. Eur Respir J 2011; 37: 472-474.

5 Lavelle A, Sugrue R, Lawler G, et al. Sitaxentan-induced hepatic failure in two patients with pulmonary arterial hypertension. Eur Respir J 2009; 34: 770-771.

6 European Medicines Agency. Thelin (sitaxentan) to be withdrawn due to cases of unpredictable serious liver injury. www.ema.europa. eu/ema/index.jsp?curl=pages/news_and_events/news/2010/12/ news_detail_001161.jsp\&murl=menus/news_and_events/news_ and_events.jsp\&mid $=$ WC0b01ac058004d5c1\&jsenabled $=$ true Date last updated: December 11, 2010. Date last accessed: December 16, 2010.

DOI: 10.1183/09031936.00194810

\section{Dyspnoea: a prognostic marker for idiopathic pulmonary fibrosis}

\section{To the Editors:}

NiSHIYAMA et al. [1] have nicely evaluated the role of dyspnoea in daily life as an independent prognostic marker in idiopathic pulmonary fibrosis (IPF). However, a few patient- and diseaserelated confounding factors should be kept in mind while evaluating this distressing symptom.

IPF is characterised by episodes of rapid deterioration secondary to acute exacerbations or infections [2], which may profoundly increase the severity of breathlessness. This feature has inadvertently not been considered by NisHIYAMA et al. [1] while prognosticating regarding patients with IPF, and has possibly led to inaccurate disease assessment. Serial evaluation of dyspnoea could have solved this issue and strengthened its prognostic role, but was not carried out during the study. In addition, factors such as age, sex and cultural background, disease complications such as pulmonary hypertension, and comorbid conditions such as asthma, anaemia, heart failure and renal failure may significantly alter dyspnoea level and lead to misinterpretation of disease survival. Severity of breathlessness in IPF may also be affected by the nutritional status (muscle mass) and level of daily activity (exercise tolerance) of the subjects, which were not discussed in this study.

Dyspnoea is a purely subjective parameter and can even be altered by a patient's state of mind. More importantly, the patient's perception of dyspnoea (and its grading) may change with involvement of other organ system or the presence of other symptoms about which the patient is more concerned.

Dyspnoea, although the most common symptom of IPF, is at times difficult to interpret. Grading of dyspnoea may provide an important clue regarding disease severity, but should never hinder a clinician from predicting disease outcome using proven objective parameters.
Serial evaluation of dyspnoea may be more appropriate than a single assessment in prognosticating IPF.

\section{Aggarwal* and P.R. Mohapatra ${ }^{\#}$}

*Pulmonary and Critical Care, Fortis Escorts Hospital, Amritsar, and "Dept of Pulmonary Medicine, Government Medical College and Hospital, Sector 32, Chandigarh, India.

Correspondence: D. Aggarwal, Gali No. 5, House No. 11-E, Hukam Singh Road, Amritsar, Punjab, India. E-mail: aggarwaldr@ yahoo.co.in

Statement of Interest: None declared.

\section{REFERENCES}

1 Nishiyama $\mathrm{O}$, Taniguchi $\mathrm{H}$, Kondoh $\mathrm{Y}$, et al. A simple assessment of dyspnoea as a prognostic indicator in idiopathic pulmonary fibrosis. Eur Respir J 2010; 36: 1067-1072.

2 Kim DS, Park JH, Park BK, et al. Acute exacerbation of idiopathic pulmonary fibrosis: frequency and clinical features. Eur Respir J 2006; 27: 143-150.

DOI: $10.1183 / 09031936.00141610$

\section{From the authors:}

We read with genuine interest the correspondence of D. Aggarwal and P.R. Mohapatra, in which they commented on our article demonstrating the relationship between daily dyspnoea and survival in idiopathic pulmonary fibrosis (IPF) [1].

Acute exacerbation of IPF is characterised by acute deterioration of dyspnoea within a few weeks, newly bilateral lung 